\title{
Has the Substructure of Quarks Been Found by the Collider Detector at Fermilab?
}

\author{
Keiichi Akama \\ Department of Physics, Saitama Medical College, Kawakado, Moroyama, Saitama 350-04, Japan \\ Hidezumi Terazawa \\ Institute for Nuclear Study, University of Tokyo, Midori-cho, Tanashi, Tokyo 188, Japan
}

\begin{abstract}
The significant excess recently found by the CDF Collaboration in the inclusive jet cross section for jet transverse energies $E_{T} \geq 200 \mathrm{GeV}$ over current QCD predictions can be explained either by possible production of excited bosons (excited gluons, weak bosons, Higgs scalars, etc.) or by that of excited quarks. The masses of the excited boson and the excited quark are estimated to be around $1600 \mathrm{GeV}$ and $500 \mathrm{GeV}$, respectively.
\end{abstract}

12.50.-d, 12.50.Ch, 12.50.Fk, 13.85.-t, 13.87.-a

The CDF Collaboration at the Fermilab Tevatron collider [1] has reported their data on the inclusive jet cross section for jet transverse energies, $E_{T}$, from 15 to $440 \mathrm{GeV}$, in the pseudorapidity region, $0.1 \leq|\eta| \leq 0.7$, with the significant excess over current predictions based on perturbative QCD calculations for $E_{T} \geq 200 \mathrm{GeV}$, which may indicate the presence of quark substructure at the compositeness energy scale, $\Lambda_{C}$, of the order of $1.6 \mathrm{TeV}$. It can be taken as an exciting and already intriguing historical discovery of the substructure of quarks (and leptons), which has been long predicted, or as the first evidence for the composite models of quarks (and leptons), which has been long proposed since the middle of 1970's 2 - 4 . Note that such relatively low energy scale for $\Lambda_{C}$ of the order of $1 \mathrm{TeV}$ has recently been anticipated rather theoretically [5] or by precise comparison between currently available experimental data and calculations in the composite models of quarks (and leptons) [6]. However, the CDF experimental observation may certainly be taken as a more direct evidence for the substructure of quarks. The purpose of this letter is to explain the observed excess either by possible production of excited bosons (excited gluons, weak bosons, Higgs scalars, etc.) or by that of excited quarks and to estimate the masses of the excited boson and the excited quark to be around 1600 $\mathrm{GeV}$ and $500 \mathrm{GeV}$, respectively.

An important motivation for composite models of quarks and leptons is to explain the repetition of generation structure in the quark and lepton spectrum. The repetition of isodoublets of quarks and leptons suggests the possible existence of an isodoublet of subquarks, $w_{i}(i=1,2)$ (called "wakems" standing for weak electromagnetic), while the repetition of color-quartets of quarks and leptons does that of a color-quartet of subquarks, $C_{\alpha}(\alpha=0,1,2,3)$ (called "chroms" standing for colors) [2]. Then, the quarks $(q)$ and leptons $(l)$ are taken as composites of subquarks including $w_{i}$ and $C_{\alpha}$. In this picture, the weak bosons $\left(W^{ \pm}\right.$and $\left.Z\right)$, the gluons $\left(G^{a}, a=1,2, \cdots, 8\right)$, the Higgs scalars $\left(\phi^{+}, \phi^{0}\right)$ [and even the photon $(\gamma)$ ], can also be taken as composites of a subquark and an antisubquark such as $w_{i}$ and $\bar{w}_{j}$ or $C_{\alpha}$ and $\bar{C}_{\beta}$. In these models, we expect that there may appear not only exotic states and excited states of the fundamental fermions but also those of the fundamental bosons [7]. Their expected properties and various effects have been studied extensively in Ref. [8]. In what follows, we shall discuss the results of our investigation on the leading-order effects of such excited quarks and bosons to the inclusive jet production cross section for $p \bar{p}$ scattering of $p \bar{p} \rightarrow$ jet + anything.

Let us first consider excited bosons or bosonic composites in more general. Let us denote the vector and color-octet, vector and color-singlet, scalar and color-octet, and scalar and color-singlet bosonic composites by $V_{\mu}^{a}, V_{\mu}, S^{a}$, and $S$, respectively. Then, the dimensionless couplings between these bosonic composites and quarks are given by the following interaction Lagrangian:

$$
\begin{aligned}
L_{\mathrm{int}} & =g_{V 8} \bar{q} \frac{1}{2} \lambda_{a} V_{\mu}^{a} \gamma^{\mu}\left(\eta_{L 8} \gamma_{L}+\eta_{R 8} \gamma_{R}\right) q+g_{S 8} \bar{q} \frac{1}{2} \lambda_{a} S^{a} q \\
& +g_{V 1} \bar{q} \frac{1}{2} \lambda_{0} V_{\mu}^{a} \gamma^{\mu}\left(\eta_{L 1} \gamma_{L}+\eta_{R 1} \gamma_{R}\right) q+g_{S 1} \bar{q} \frac{1}{2} \lambda_{0} S q
\end{aligned}
$$

where $\gamma_{L}=\left(1-\gamma_{5}\right) / 2, \gamma_{R}=\left(1+\gamma_{5}\right) / 2, g_{V 8}, g_{S 8}, g_{V 1}, g_{S 1}$ are coupling constants, $\lambda_{a}(a=1,2, \cdots, 8)$ are the Gell-Mann matrices for color $\mathrm{SU}(3), \lambda_{0}$ is the $\sqrt{2 / 3}$ times $3 \times 3$ unit matrix, and $\left(\eta_{L 8}, \eta_{R 8}\right)$ or $\left(\eta_{L 1}, \eta_{R 1}\right)=(1,1),(1,-1),(1,0)$, and $(0,1)$ for the vector, axial vector, left-handed, and right-handed couplings, respectively. $V_{\mu}^{a}$ and $V_{\mu}$ are hermitian fields while $S^{a}$ and $S$ are in general complex. These interactions respect the chiral symmetry of quarks. Note that the dimensionless coupling between gluons, $G^{a}$, and $V^{a}$ must have a form of $G^{a \mu \nu}\left(D_{\mu} V_{\nu}^{a}-D_{\nu} V_{\mu}^{a}\right)$ and, therefore, have no physical effect since it can be absorbed into the kinetic term of $\left(G_{\mu \nu}^{a}\right)^{2}$ after diagonalizing of $G^{a}$ and $V^{a}$. 
Also note that there exist no dimensionless couplings of $G^{a}$ and $V, G^{a}$ and $S^{a}$, or $G^{a}$ and $S$. Therefore, these bosonic composites contribute to $p \bar{p}$ scatterings only through $q \bar{q} \rightarrow q \bar{q}$ scatterings and their crossed channels.

Let $(s, t, u)$ and $z$ be the Mandelstam variables for the elementary process of $q \bar{q} \rightarrow q \bar{q}$ scattering and $\cos \theta$ with the scattering angle $\theta$ in the center-of-mass system. Then, the differential cross section for the scattering is given by

$$
\frac{d \sigma}{d z}=\frac{1}{36} \cdot \frac{1}{32 \pi s}\left[A_{L}(s, t, u)+A_{R}(s, t, u)+2 B(s, t, u)+2 B(t, s, u)\right]
$$

where

$$
\begin{gathered}
A_{x}(s, t, u)=4 u^{2}\left\{2\left|V_{8}^{x x}(s)\right|^{2}+2\left|V_{8}^{x x}(t)\right|^{2}+9\left|V_{1}^{x x}(s)\right|^{2}+9\left|V_{1}^{x x}(t)\right|^{2}\right. \\
+2 R e\left[-\frac{2}{3} V_{8}^{x x}(s)^{*} V_{8}^{x x}(t)+4 V_{8}^{x x}(s)^{*} V_{1}^{x x}(t)\right. \\
\left.\left.+4 V_{1}^{x x}(s)^{*} V_{8}^{x x}(t)+3 V_{1}^{x x}(s)^{*} V_{1}^{x x}(t)\right]\right\}, \quad(x=L, R) \\
B(s, t, u)=t^{2}\left\{4\left[2\left|V_{8}^{L R}(s)\right|^{2}+9\left|V_{1}^{L R}(s)\right|^{2}\right]+t^{2}\left[2\left|S_{8}(t)\right|^{2}+9\left|S_{1}(t)\right|^{2}\right]\right. \\
-4 R e\left[-\frac{2}{3} V_{8}^{L R}(s)^{*} S_{8}(t)+4 V_{8}^{L R}(s)^{*} S_{1}(t)\right. \\
\left.\left.+4 V_{1}^{L R}(s)^{*} S_{8}(t)+3 V_{1}^{L R}(s)^{*} S_{1}(t)\right]\right\}
\end{gathered}
$$

with the propagators

$$
\begin{aligned}
& V_{1}^{x y}(s)= \begin{cases}\frac{e^{2}}{s}+\frac{g_{Z x} g_{Z y}}{s-M_{Z}^{2}+i M_{Z} \Gamma_{Z}}+\frac{g_{V 1}^{2} \eta_{x 1} \eta_{y 1}}{s-M_{V 1}^{2}+i M_{V 1} \Gamma_{V 1}}, & (x, y=L, R) \\
\frac{g_{W} g_{W}^{\prime}}{s-M_{W}^{2}+i M_{W} \Gamma_{W}}, & (x, y=L)\end{cases} \\
& V_{8}^{x y}(s)=\frac{g^{2}}{s}+\frac{g_{V 8}^{2} \eta_{x 8} \eta_{y 8}}{s-M_{V 8}^{2}+i M_{V 8} \Gamma_{V 8}},(x, y=L, R) \\
& S_{1}(s)=\frac{g_{S 1}^{2}}{s-M_{S 1}^{2}+i M_{S 1} \Gamma_{S 1}}, \\
& S_{8}(s)=\frac{g_{S 8}^{2}}{s-M_{S 8}^{2}+i M_{S 8} \Gamma_{S 8}} .
\end{aligned}
$$

Here, $e$ is the electromagnetic coupling constant, $g$ is the QCD coupling constant, $g_{Z L}$ and $g_{Z R}$ are the left- and right-handed coupling constants of $Z$ boson, $g_{W}$ is the weak gauge coupling constant times the relevant CKM matrix element, $M_{X}$ is the mass of particle $X$, and $\Gamma_{X}$ is the decay width. If the decay of the excited boson is dominated by the two body decay due to the interactions given in Eq. (11), its decay width is given by

$$
\begin{aligned}
& \Gamma_{V 8}=\Gamma_{V 1}=\frac{M_{V}}{48 \pi} \sum g_{V}^{2} \sqrt{1-\frac{4 m^{2}}{M_{V}^{2}}}\left[\left(1-\frac{m^{2}}{M_{V}^{2}}\right)\left(\eta_{L}^{2}+\eta_{R}^{2}\right)+\frac{6 m^{2}}{M_{V}^{2}} \eta_{L} \eta_{R}\right], \\
& \Gamma_{S 8}=\Gamma_{S 1}=\frac{M_{S}}{48 \pi} \sum g_{S}^{2} \sqrt{1-\frac{4 m^{2}}{M_{S}^{2}}}\left(1-\frac{2 m^{2}}{M_{S}^{2}}\right),
\end{aligned}
$$

where $\sum$ denotes the summation over flavors of final quarks and $m$ 's are the final quark masses, all of which but the top quark mass can be practically neglected.

Let us next consider excited quarks (of spin 1/2 for simplicity), which are denoted by $Q$ 's. Then, the interaction of $Q$ with quarks $(q)$ and gluons $\left(G_{\mu}^{a}\right)$ is given by

$$
L_{i n t}=-\frac{g_{Q}}{2 M_{Q}}\left[\bar{Q} \frac{1}{2} \lambda^{a} \sigma^{\mu \nu} G_{\mu \nu}^{a} q_{L}+\overline{q_{L}} \frac{1}{2} \lambda^{a} \sigma^{\mu \nu} G_{\mu \nu}^{a} Q+(L \leftrightarrow R)\right]
$$


where $g_{Q}$ is a coupling constant and $M_{Q}$ is the excited quark mass. Note that an excited quark coupling with $q_{L}$ and another excited quark coupling with $q_{R}$ must be different from one another if the chiral symmetry of quarks is preserved. If this is the case, the differential cross section for the scattering of $q \bar{q} \rightarrow G G$ is given by

$$
\begin{aligned}
\frac{d \sigma}{d z}=\frac{1}{27 \pi s}[ & g^{4}\left(t^{2}+u^{2}\right)\left(\frac{1}{t u}-\frac{9}{4 s^{2}}\right) \\
& +\frac{g^{2} g_{Q}^{2}}{4 M_{Q}^{2}} \operatorname{Re}\left(\frac{t^{2}}{t-M_{Q}^{2}+i M_{Q} \Gamma_{Q}}+(t \leftrightarrow u)\right) \\
& \left.+\frac{g_{Q}^{4} u t}{M_{Q}^{4}}\left(\left|\frac{t}{t-M_{Q}^{2}+i M_{Q} \Gamma_{Q}}\right|^{2}+(t \leftrightarrow u)\right)\right] .
\end{aligned}
$$

If the decay of $Q$ is dominated by the interactions given in Eq. (11), the decay width of $Q$ is given by

$$
\Gamma_{Q}=\frac{g_{Q}^{2}}{6 \pi} M_{Q}\left(1-\frac{m^{2}}{M_{Q}^{2}}\right),
$$

where $m$ is the final quark mass, any one of which except the top quark mass can be practically neglected. Note that if an excited quark coupling with $q_{L}$ and another excited quark coupling with $q_{R}$ are not discriminated against one another, which leads to breaking of the chiral symmetry of quarks, the above differential cross section would need an additional term,

$$
\begin{aligned}
& \frac{1}{27 \pi s} \frac{g_{Q}^{4} u t}{M_{Q}^{4}}\left[\left|\frac{t}{t-M_{Q}^{2}+i M_{Q} \Gamma_{Q}}\right|^{2}+(t \leftrightarrow u)\right. \\
&\left.-\frac{4}{3} \operatorname{Re}\left(\frac{t u}{\left(t-M_{Q}^{2}+i M_{Q} \Gamma_{Q}\right)^{*}\left(u-M_{Q}^{2}+i M_{Q} \Gamma_{Q}\right)}\right)\right]
\end{aligned}
$$

In this case, the decay width of $Q$ would also be changed to be twice as much as given in Eq. (13). Note also that the differential cross sections for the crossed channels can easily be obtained by exchanging $(s, t, u)$ appropriately and by rewriting the statistical factors due to the different spins and colors of initial (and final) quarks (or gluons).

Now we evaluate the single jet $p_{T}$ inclusive distribution, dijet invariant mass distribution, and dijet angular distribution in the $p \bar{p}$ scattering in the CDF energy region. For the elementary processes, we take $2 \rightarrow 2$ processes of quarks, antiquarks, and gluons. Also, we assume that either one of $u, d, s, c, b$ quarks or gluons in the final states is to be observed as a jet. Although the authors of Ref. [1] have found the excess at high $p_{T}$ in comparison of their data with the next-to-leading order calculations, we have restricted ourselves to the leading order contribution from composite models. Since higher order corrections are supposed to contribute almost equally both in the standard model calculations and in the composite model ones, the ratio of the composite model calculation to the standard model one may not be so much affected by higher order corrections and may be enough meaningful even if both of the calculations are only in the leading order. As for the parton distribution functions we use those of Glück-Reya-Vogt in Ref. [9].

In FIG. 1, the predictions of the composite models with excited states for the single jet $E_{T}$ inclusive distribution divided by those of the standard model are compared with the corresponding CDF experimental result reported in Ref. [1]. We have taken the same average over the pseudorapidity range of $0.1 \leq|\eta| \leq 0.7$ as the CDF experiment [1]. Based on such comparison, we have performed detailed chi-square anlyses, and determined the allowed regions (95\% confidence level) of the mass $M_{X}$ and coupling constant $\alpha_{X}\left(\equiv g_{X}^{2} / 4 \pi\right)$ of various types of excited states $X$ (See FIG. 2). It indicates that the excited bosons with $\alpha_{X}>0.1$ and $M_{X}>1000 \mathrm{GeV}$ are allowed. The excess of the $E_{T}$ distribution is well fitted by the tail of the high mass resonance of the excited boson. On the other hand, there is no allowed region for a single excited quark, as far as we assume the two-jet decay mode dominates the decay. This is because the width (13) is too narrow to fit the rather gentle slope of the observed data in FIG. 1. The width, however, can be broadened due to (i) other decay modes such as multi-jet or semi-jet processes, (ii) coexistence of several resonances, or (iii) limited resolution for the jet energy and momentum measurement. Let $r$ be the ratio of the total decay width to the partial width (13) of the decay to the two-jet mode. In FIG. 2, we also show the allowed region for the $M_{X}$ and $\alpha_{X}$ of the excited quarks for the cases of $r=2$ and 3. It is restricted in the low-mass region $400 \mathrm{GeV}<M_{X}<900 \mathrm{GeV}$ and $0.03<\alpha_{X}<0.8$.

To get more precise information, it may be extremely useful to investigate the dijet invariant mass and angular distributions. Figure 3 shows the predictions with the typical excited states for the dijet invariant mass $\left(E_{\text {dijet }}\right)$ 
distribution divided by those of the standard model. It predicts a significant excess in the high dijet mass region. Figure 4(a) shows the predicted dijet angular distribution as a function of $\chi \equiv(1+\cos \theta) /(1-\cos \theta)$ (normalized by the average over the region of $1 \leq \chi \leq 5$ ). The model with excited states predicts relative excess in low $\chi$ (i. e. large $\theta$ ) region, since the peak at $\theta=0$ due to exchange of light quarks and massless gluons is absent in the additional contributions from the excited state. To see it more clearly, we show in FIG. 4(b) the ratio of the number of the expected events for $\chi<2.5$ to that for $\chi>2.5$ as a function of the dijet invariant mass $E_{\text {dijet }}$.

To sum up, we have shown in this letter that the significant excess found by the CDF Collaboration can be explained either by possible production of excited bosons whose masses are around $1600 \mathrm{GeV}$ or by that of excited quarks whose masses are around $500 \mathrm{GeV}$. The copious production of such excited particles can be expected in the future $e^{+} e^{-}$NLC experiments and $p \bar{p}$ LHC experiments. In conclusion, we must mention that although we have assumed the excited quarks of spin $1 / 2$ for simplicity, one can also assume those of spin $3 / 2$, which has very recently been emphasized by Bander [10].

\section{ACKNOWLEDGEMENTS}

The authors would like to thank Professor Kunitaka Kondo for giving them the valuable information on the CDF experiment and for sending them the manuscripts before publication. One of the authors (H.T.) also wishes to thank Professor Stanley J. Brodsky and all the other staff members, especially Professors James D. Bjorken and Michael Peskin, of Theoretical Physics Group at Stanford Linear Accelerator Center, Stanford University not only for their useful discussions on the substructure of quarks but also for their warm hospitalities extended to him during his visit in July, 1996 when this work was completed. The other author (K.A) also wishes to thank Professors Yuichi Chikashige and Tadashi Kon for useful discussions and communications.

[1] CDF Collaboration, F. Abe et al., Phys. Rev. Lett. $\underline{77}, 438$ (1996).

[2] J.C. Pati and A. Salam, Phys. Rev. D $\underline{10}, 275$ (1974); H. Terazawa, Y. Chikashige, and K. Akama, Phys. Rev. D 15 , 480 (1977); H. Terazawa, Phys. Rev. D $\underline{22}, 184$ (1980).

[3] For a review, see, for example, H. Terazawa, in Proc. XXII International Conference on High Energy Physics, Leipzig, 1984, edited by A. Meyer and E. Wieczorek (Akademie der Wissenschaften der DDR, Zeuthen, 1984), Vol. I, p. 63.

[4] For a recent review, see, for example, H. Terazawa, in Proc. of the Fourth International Conference on Squeezed States and Uncertainty Relations, Taiyuan, Shanxi, China, 1995, edited by D. Han et al., NASA Conference Publication $\underline{3322}$ (NASA Goddard Space Flight Center, Greenbelt, Maryland, 1996), p. 179.

[5] See, for examples, H. Terazawa, Phys. Rev. Lett. $\underline{65}, 823$ (1990); K. Akama, H. Terazawa, and M. Yasuè, Phys. Rev. Lett. $\underline{68}$, 1826 (1992).

[6] See, for example, K. Akama and H. Terazawa, Phys. Lett. B321, 145 (1994); Mod. Phys. Lett. A99, 3423 (1994); H. Terazawa, Institute for Nuclear Study, University of Tokyo, Tokyo, Report No. INS-Rep.-1131, Jan. 1996.

[7] H. Terazawa, in Ref. [2].

[8] K. Akama and T. Hattori, Phys. Rev. D $\underline{40}, 3688$ (1989); K. Akama, T. Hattori, and M. Yasuè, Phys. Rev. D $\underline{42}, 789$ (1990); D $\underline{43}, 1702$ (1991); S. Ishida and M. Sekiguchi, Prog. Theor. Phys. $\underline{86}$, 491 (1991); K. Akama and T. Hattori, Int. J. Mod. Phys. A99, 3503 (1994).

[9] M. Glück, E. Reya, and A. Vogt, Z. Phys. C67, 433 (1995).

[10] M. Bander, Phys. Rev. Lett. 77, 601 (1996). 


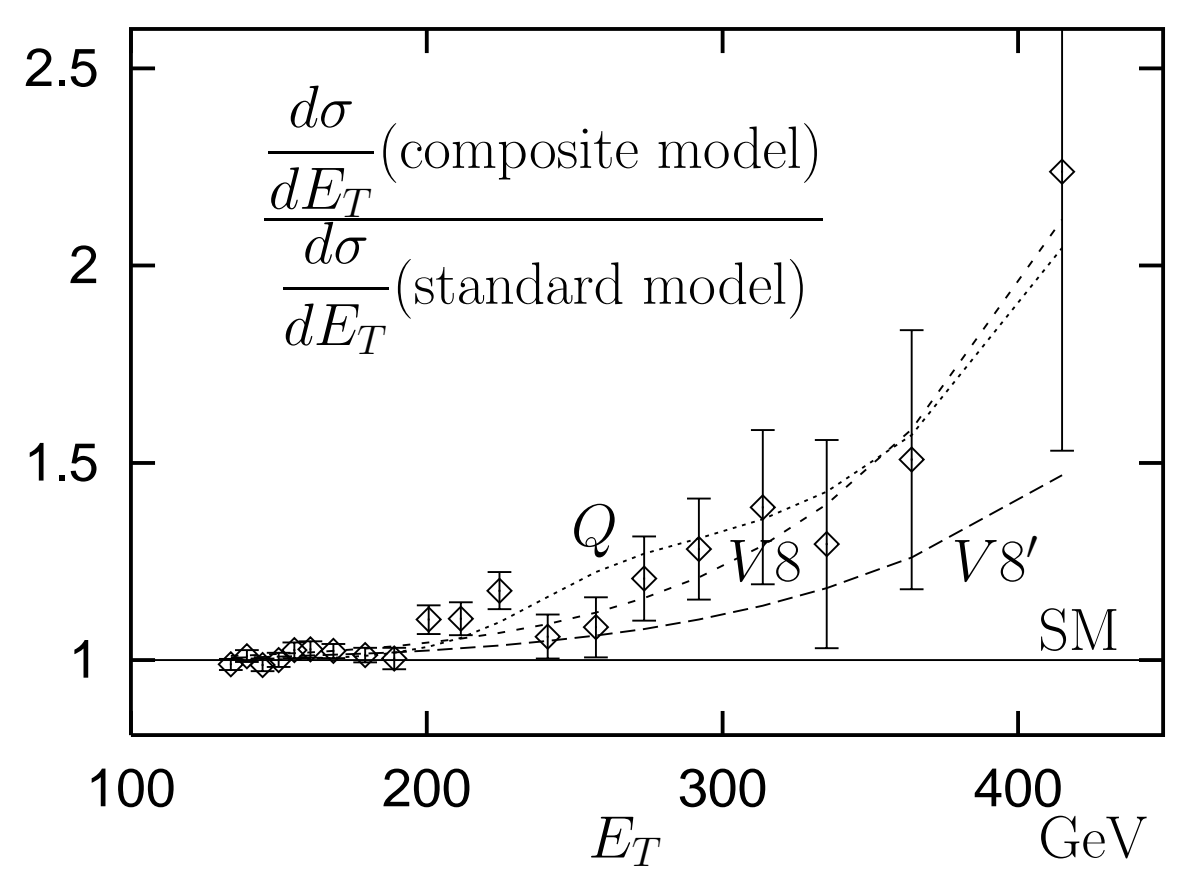

FIG. 1. The predictions of the composite models with excited states for the single jet $E_{T}$ inclusive distribution divided by those of the standard model. The label SM indicates the prediction of the standard model, $V 8\left(V 8^{\prime}\right)$ indicates that with a vector octet excited boson with $M_{V 8}=1600 \mathrm{GeV}(2000 \mathrm{GeV})$ and $\alpha_{V 8}=g_{V 8}^{2} / 4 \pi=1$, and $Q$ indicates that with the excited quark with $M_{Q}=500 \mathrm{GeV}, \alpha_{Q}=g_{Q}^{2} / 4 \pi=0.2$, and $r=3$, where $r$ is the ratio of the decay width to the partial width of the decay to the two-jet mode. The points with an error bar are the corresponding CDF experimental results reported in Ref. [1].

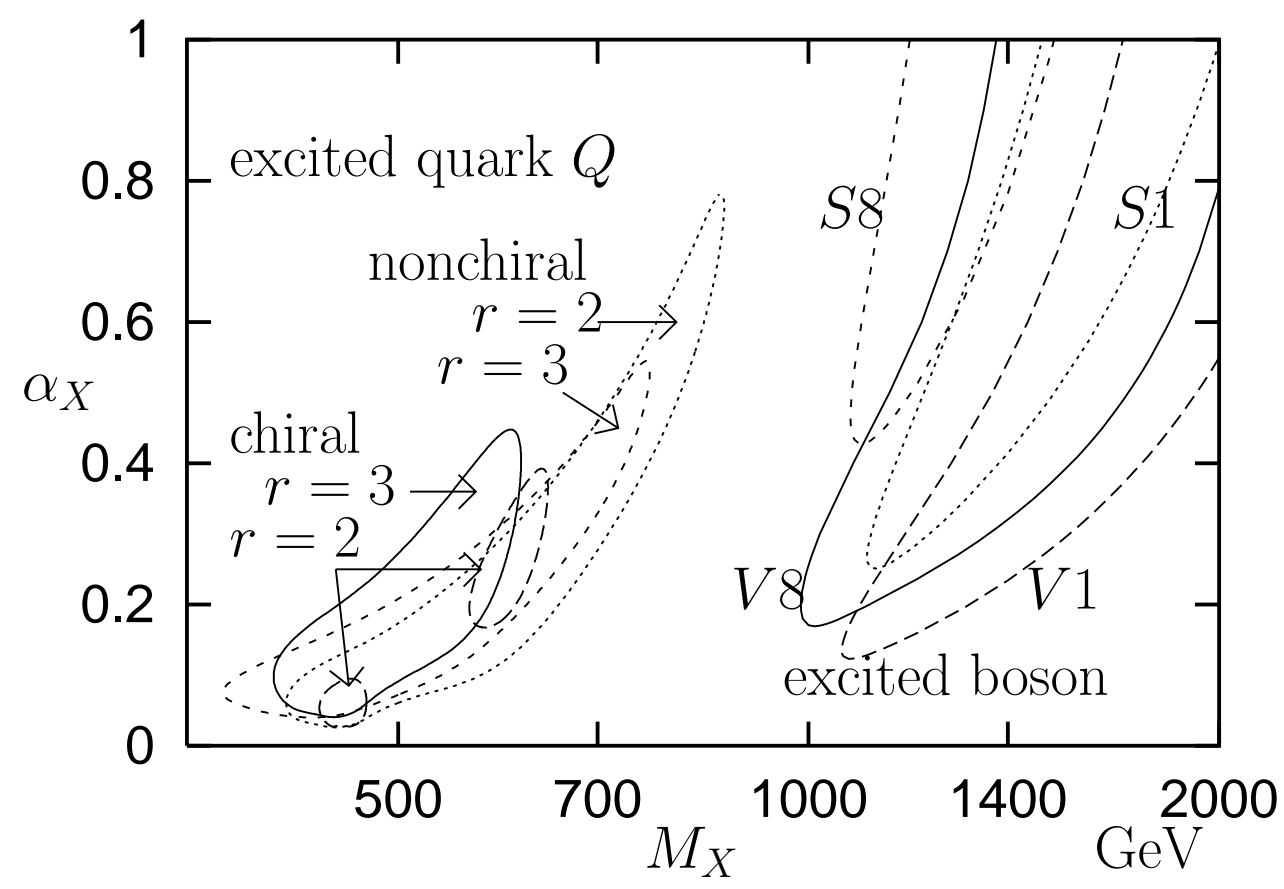

FIG. 2. The allowed regions (95\% confidence level) of the mass $M_{X}$ and coupling constant $\alpha_{X}$ (三 $\left.g_{X}^{2} / 4 \pi\right)$ of various types of excited states $X$. The labels $V 8, V 1, S 8$, and $S 1$ indicate vector-octet, vector-singlet, scalar-octet, and scalar-singlet excited bosons, respectively. 


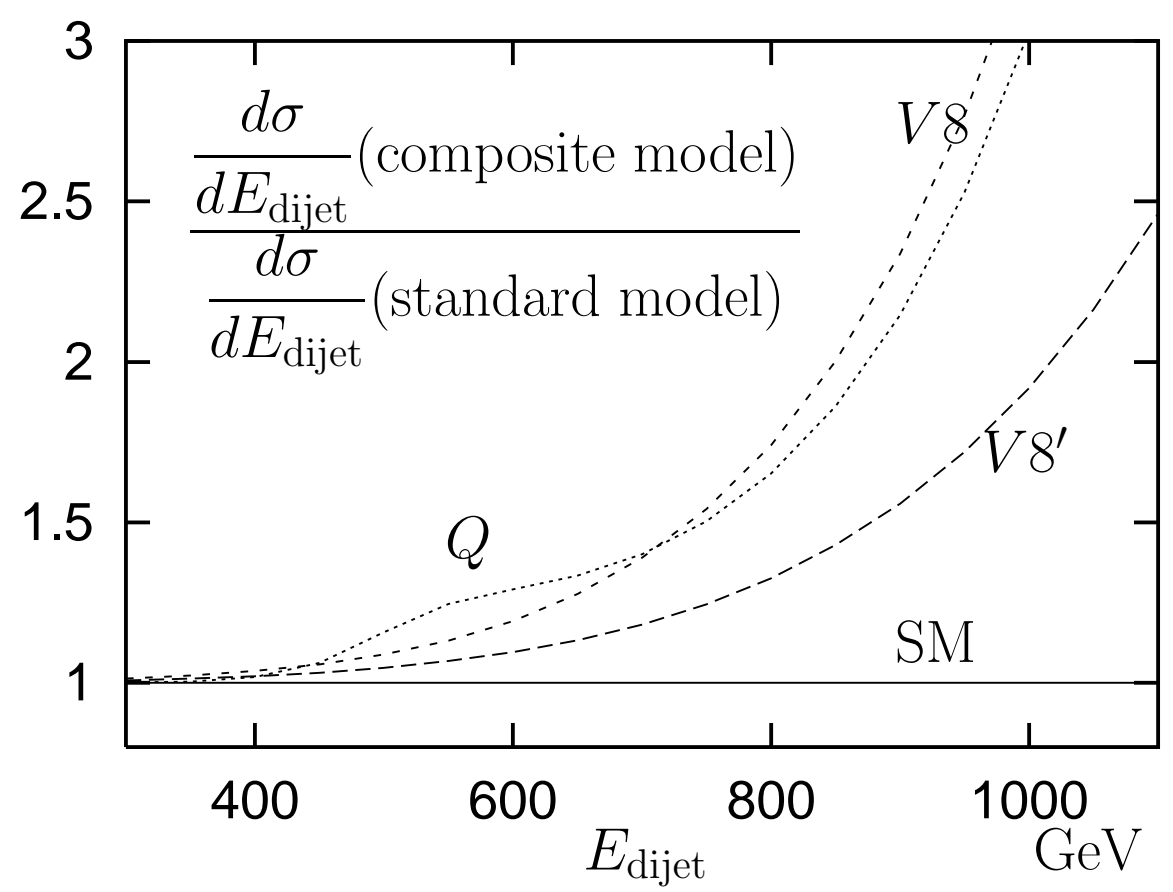

FIG. 3. The predictions with the typical excited state for the dijet invariant mass $\left(=E_{\text {dijet }}\right)$ distribution divided by those of the standard model. The labels SM, $V 8, V 8^{\prime}$, and $Q$ are the same as those in FIG. 1.
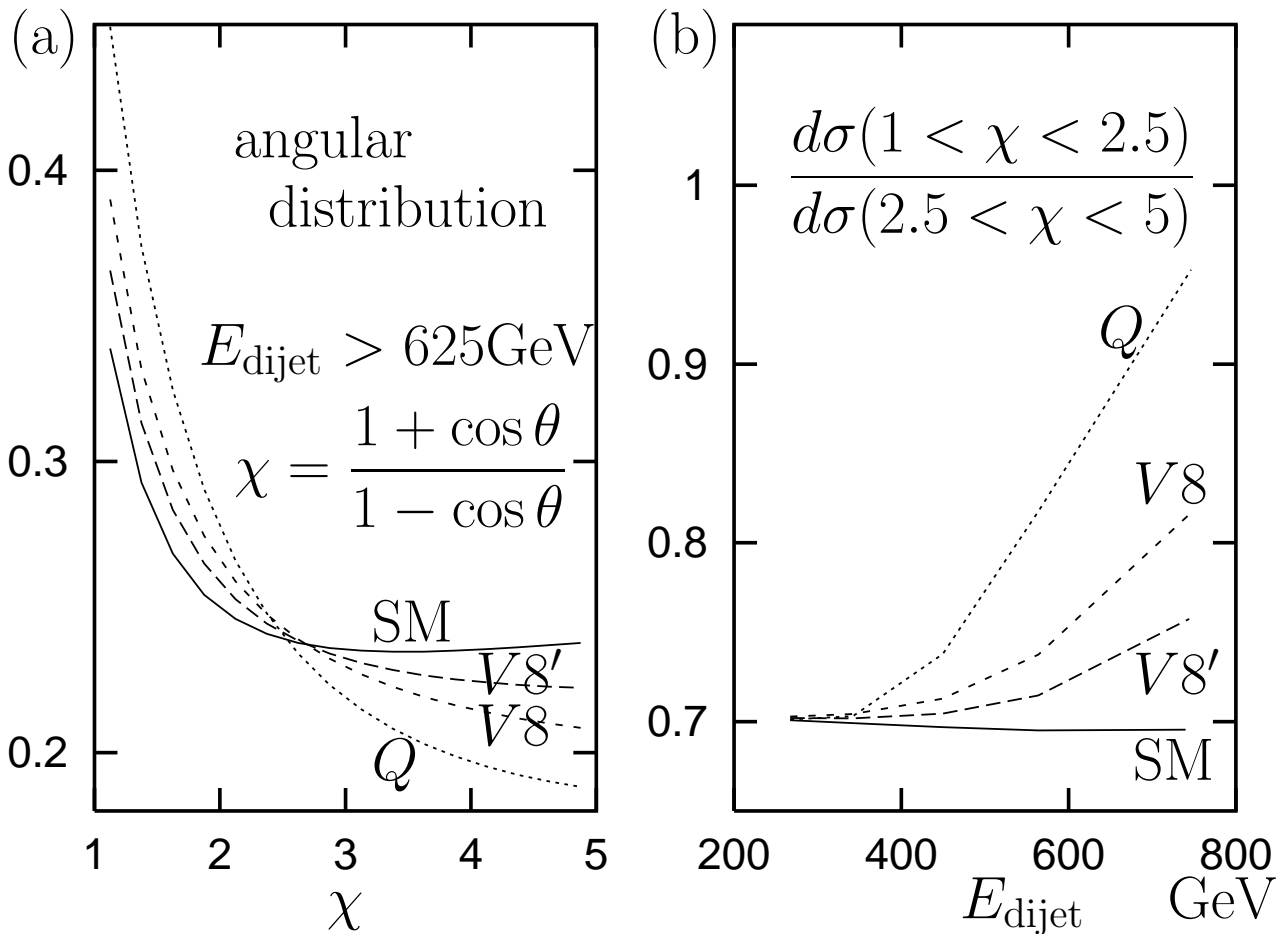

FIG. 4. (a) The predicted dijet angular distribution as a function of $\chi$ normalized by the average over the region of $1 \leq \chi \leq 5$. (b) The ratio of the number of the expected events for $\chi<2.5$ to that for $\chi>2.5$ as a function of the dijet invariant mass $E_{\text {dijet }}$. The labels SM, $V 8, V 8^{\prime}$, and $Q$ are the same as those in FIG. 1. 